\title{
Contratación pública socialmente responsable
}

\author{
Socially responsible public procurement
}

\author{
Fernando Juárez-Urquijo
}

Juárez-Urquijo, Fernando (2021). "Contratación pública socialmente responsable". Anuario ThinkEPI, v. 15, e15a03.

https://doi.org/10.3145/thinkepi.2021.e15a03

\section{Fernando Juárez-Urquijo}

https://orcid.org/0000-0002-8466-4511

\author{
Biblioteca Municipal de Muskiz \\ C/ Cendeja 29. 48550, Muskiz, Bizkaia (España) \\ ferjur@gmail.com
}

Publicado en IweTel el 14 de septiembre de 2021

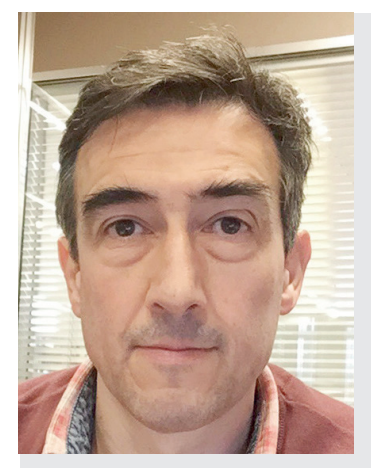

\begin{abstract}
Resumen: Estamos a las puertas de un relevo generacional en los servicios GLAM públicos (bibliotecas, archivos, museos, centros de documentación) y el panorama que nos adelantan los actuales modelos de contratación auguran un aumento del número de externalizaciones. Es previsible la incorporación de una cohorte de nuevos profesionales que trabajarán para las diferentes administraciones sin ser empleados públicos y realizando, en muchas ocasiones, labores de carácter permanente. Esta nota se va a centrar en la problemática de la contratación pública vía externalización, en la oportunidad que, para promover la sostenibilidad, la equidad y la innovación, tienen las administraciones públicas si utilizan su gran "poder de compra" con criterios de responsabilidad social y en el papel de las asociaciones profesionales en la regulación de nuevos ecosistemas laborales.
\end{abstract}

Palabras clave: Servicios GLAM; Modelos de gestión; Contrataciones; Marco laboral; Asociaciones profesionales.

\begin{abstract}
We are on the verge of a generational changeover in public GLAM services (libraries, archives, museums, documentation centers), and predictions based on current contracting models indicate an increase in the amount of outsourcing. It is foreseeable that a cohort of new professionals will join the various administrations without being public employees, often carrying out permanent tasks. This note focuses on the problems of public procurement through outsourcing, on the opportunity for public administrations to promote sustainability, equity, and innovation if they use their great "purchasing power" with social responsibility criteria, and on the role of professional associations in the regulation of new labor ecosystems.
\end{abstract}

Keywords: GLAM services; Management models; Hiring; Work framework; Professional associations.

\section{Preámbulo}

Comentábamos en una nota de Thinkepi anterior (Juárez-Urquijo, 2019) que estamos a las puertas de un relevo generacional en los servicios GLAM públicos (bibliotecas, archivos, museos, centros de documentación) y que el panorama que nos adelantan los actuales modelos de contratación augura un aumento del número de externalizaciones. Es previsible la incorporación de una cohorte de nuevos profesionales que trabajarán para las diferentes administraciones sin ser empleados públicos y realizando, en muchas ocasiones, labores de carácter permanente.

Esta nota se va a centrar en la problemática de la contratación pública vía externalización, en la oportunidad que, para promover la sostenibilidad, la equidad y la innovación, tienen las administraciones públicas si utilizan su gran "poder de compra" con criterios de responsabilidad social y en el papel de las asociaciones profesionales en la regulación de nuevos ecosistemas laborales. 


\section{2. ¿Hablamos?}

En la recepción inaugural de las Jornadas Españolas de Información y Documentación 2019 (JEID19)1 organizadas por Fesabid (Federación Española de Sociedades de Archivística, Biblioteconomía, Documentación y Museística), una persona se acercó para preguntarme si era el representante de Aldee (Asociación Vasca de Profesionales de Archivos, Bibliotecas y Centros de Documentación) (acababa de estrenarme como presidente de la asociación).

https://www.fesabid.org

https://www.aldee.eus/es

Tras los saludos protocolarios hablamos distendidamente sobre nuestro (común) origen geográfico, los conocidos que compartíamos y sobre nuestras circunstancias laborales, ella con cargo de responsabilidad en una empresa privada dedicada a la gestión de archivos y centros de documentación, sobre todo públicos, yo funcionario en la administración local trabajando en una biblioteca pública. Tras unos minutos la conversación llegó al punto buscado: en su empresa estaban preocupados porque no eran capaces de retener el capital humano (personas en las que invertían tiempo y dinero para mejorar sus conocimientos y habilidades pero que abandonaban la empresa buscando mejores ofertas laborales, casi siempre de las administraciones) y tenían problemas para encontrar relevo cualificado; se preguntaba si nuestra asociación podría ayudar orientando la formación hacia lo que el mercado (empresas y administraciones) demandaba. En ese punto comenté que, en mi opinión, su problema no era tanto de formación como de condiciones laborales y que desaparecería, en gran medida, con un aumento de sueldos. Ante mi simpleza ("Sí, claro, es evidente, pero hay que tener en cuenta las reglas del juego") mi interlocutora decidió impartir un cursillo acelerado sobre licitaciones públicas.

\section{3. ¿A cuánto están los panes y los peces?}

En las administraciones tenemos menos personal que hace treinta años a pesar de que ofertamos una mayor cantidad de servicios. ¿Panes y peces? No, el milagro se llama subcontratación de servicios y se está generalizando: los "compradores públicos" son los principales inversores en Europa y sus gastos representan ya el $14 \%$ del producto interior bruto de la UE. Se presupone que a través de la externalización las administraciones mejoran su eficiencia, son más eficaces y reducen costes, pero lo cierto es que sabemos poco:

- sobre la valoración que los ciudadanos hacen de la provisión de los servicios públicos y sus preferencias en cuanto a la prestación de los mismos por un ente público o privado;

- sobre el grado de externalización de los servicios públicos que se ha alcanzado en España en la actualidad;

- sobre las condiciones en que las administraciones públicas garantizan la calidad de los servicios externalizados a través de su seguimiento y control;

- sobre los costes socioeconómicos a medio y largo plazo.

Mediante el uso estratégico de la externalización las administraciones aprovechan las capacidades de innovación de los proveedores para delegar la gestión de servicios no esenciales y enfocar esfuerzos a sus actividades nucleares. Se contratan sobre todo servicios que afectan a tareas que son intensivas en recursos humanos que no requieren alta cualificación, las altamente especializadas (bibliotecas, archivos, centros de documentación y museos funcionan gracias a profesionales cualificados), o las que están sujetas a cargas de trabajo que pueden ser fluctuantes y finalistas. Para elegir la mejor oferta se establecen unos criterios técnicos (cuya valoración depende de un juicio de valor), unas condiciones económicas (evaluables mediante la utilización de fórmulas) y unos mecanismos de ponderación que, sistemáticamente, otorgan más peso a los aspectos económicos. Si una empresa quiere ser competitiva tiene que rebajar sus costes y eso se consigue reduciendo trabajadores y salarios. 


\section{Mejorar servicios recortando gastos}

Mejorar servicios recortando gastos: oxímoron perfecto. Esta manera de entender y aplicar la gestión pública buscando la flexibilidad y la eficacia incidiendo sistemáticamente en un recorte del gasto conduce, sin remedio, a:

- minusvalorar las condiciones técnicas frente a las económicas (algo muy sensible cuando se realizan tareas especializadas);

- la precariedad laboral y el descontento profesional;

- la fuga de profesionales que dejarán las empresas en busca de mejores condiciones;

- surgimiento de conflictos para corregir situaciones (sobre todo donde haya un mínimo de masa laboral que abra la puerta a la acción sindical);

- la preocupación de ciudadanos y administraciones por la discontinuidad en los servicios.

Sí, el recorte de gastos a corto plazo se consigue, pero sobre la mejora del servicio hay dudas razonables.

En su intento de mejorar ahorrando las administraciones están, sin pretenderlo, pauperizando profesionales y empresas, generando tensiones laborales, empeorando servicios y dañando el ecosistema informacional. La demanda que se planteó en el cóctel iba sobre formación en un entorno de alta volatilidad laboral, la respuesta que desde el mundo asociativo estamos buscando pasa por entender cómo funcionan los mecanismos de contratación para proponer y propiciar unas condiciones de externalización que beneficien a todas las partes implicadas.

\section{Externalización e igualdad de condiciones: un poco de (cruda) realidad}

Para elegir proveedores se establecen procesos de "compra" que garantizan la concurrencia empresarial en igualdad de condiciones. En los pliegos de licitación se recogen, de forma pormenorizada, tanto la descripción detallada de las condiciones de prestación y de las obligaciones que asume el contratista, como, muy importante, las prerrogativas de la administración (interpretación, resolución, etc.), que actúan como garantes de la correcta prestación del objeto de cada contrato. Al menos en teoría gana el contrato la mejor oferta: aquella que ofrece un buen equilibrio entre calidad y precio.

Quien externaliza matiza aspectos técnicos (exige perfiles, titulaciones, servicios a cubrir, horarios...) y económicos (establece el techo de gasto tras calcular un importe que garantiza margen de beneficio empresarial y pago de salarios que, sin igualar los que perciben "los trabajadores de la casa", cumplen todos los requisitos del mercado laboral) remitiendo al "convenio del sector" para regular el marco laboral a aplicar. La inexistencia de un convenio propio en nuestro sector deja abierta, de manera legal, la posibilidad a que las empresas compitan en una licitación con marcos laborales diferentes (oficinas y despachos, ocio, intervención social, artes gráficas...). Si, además, no se establecen puntuaciones mínimas en el apartado técnico ni se limitan los porcentajes de rebaja en el precio, el resultado es que el actual mecanismo favorece a las empresas "más baratas". Ser barato se consigue adelgazando márgenes de beneficio, gastos de gestión y, sobre todo, un gasto de personal condicionado por el convenio laboral a aplicar. Esta discrecionalidad condiciona las ofertas, desvirtúa las valoraciones y permite las desigualdades.

En una Resolución del Defensor del Pueblo Andaluz (Andalucía, 2017) formulada en la queja 15/5470 dirigida a la Consejería de Hacienda y Administración Pública se afirma que la externalización de la acción administrativa no es objeto de sopesado análisis previo a la adopción de la decisión sobre la misma, así como que tampoco es producto de una planificación y diseño en su implementación y que, ciertamente, es deficitaria en su fase de seguimiento (control y evaluación), circunstancia que contrasta con el amplio uso que de esta figura se hace por parte de los distintos entes que conforman el sector público, de amplia presencia en los diversos ámbitos de la acción pública (sanidad, educación, servicios sociales, obras públicas, protección civil, etc.). Las administraciones carecen de un modelo de externalización que estandarice el proceso de esta técnica de gestión en sus sucesivas fases de adopción, implementación y seguimiento.

Puede suceder, y sucede (Euskadi, 2021), que una administración generosa a la hora de dotar económicamente una licitación (consignando unas cantidades que cubran gastos de gestión, permitan beneficio empresarial y salario digno) pero ¿descuidada? en la redacción de los mecanismos de ponderación facilite que una empresa que en el apartado técnico obtenga 14,75 sobre 40 puntos (un 3,6 sobre 10) consiga superar a otra empresa que sea valorada técnicamente con un 32 sobre 40 (8 puntos) gracias a una reducción en la oferta económica del $40 \%$ sobre la cantidad que la administración considera correcta para realizar el servicio (un $30 \%$ más barata que su rival). Es probable que las personas propuestas por ambas empresas estén bien cualificadas para desempeñar el trabajo, aunque, obviamente, lo que percibirán a final de mes sea sustancialmente diferente. Sí, la administración ha conseguido ahorrar optando 
por una contratación que sus propios técnicos han calificado como deficiente ( 3,6 sobre 10), premiando a una empresa que paga peor a sus trabajadores (aunque es cierto que aplica un convenio laboral legal) y desestimando, por poco "competitivas", empresas que con una valoración técnica superior intentan mejorar las condiciones salariales de sus profesionales (con convenios laborales más favorables).

\section{Saber hacer}

Para garantizar la viabilidad y sostenibilidad de los servicios públicos las administraciones buscan, en teoría, el saber hacer de la iniciativa privada; en la práctica las actuales políticas de externalización penalizan a las empresas que cuidan el know how (estableciendo condiciones laborales que permitan retener a esos profesionales especializados e invertir en su formación) y premia a empresas con estrategias de bajo precio.

El detonante de los 229 días de huelga de Ubik (Tabakalera) en 2019 se encuentra en unos pliegos de condiciones que permitieron situaciones como la descrita. Una de las soluciones aplicadas para reconducir la situación (Tabakalera, 2019) fue volver a licitar el servicio asumiendo las mejoras laborales pactadas entre los trabajadores y la empresa adjudicataria (incremento salarial del 32,3\%, una jornada inferior a la que desempeñaban antes del conflicto, mejoras en la relación con los turnos de trabajo, especialmente los fines de semana) y articulando mecanismos -se estableció un convenio de referencia al que tendrían que ceñirse las empresas- para evitar los problemas derivados de la falta de un convenio sectorial propio (Euskadi, 2019).

Preocupa que una falla detectada y solucionada en 2019 se replique en 2021 en el mismo territorio y por alguna de las administraciones implicadas. Desde las asociaciones creemos importante:

- alertar a las administraciones sobre los efectos de la falta de un mecanismo que garantice licitar en igualdad de condiciones;

- proponer que se aplique un "convenio de referencia de mínimos" mientras se consigue la creación de un convenio propio;

- hablar con sindicatos y empresas del sector para que entablen las negociaciones necesarias para redactar y aprobar un convenio que tenga en cuenta las peculiaridades de los servicios GLAM.

\section{El gran problema: falta de músculo para implantar un marco regulatorio sectorial}

Los convenios laborales regulan aspectos laborales básicos en materia de funciones, horarios, retribuciones, conciliación familiar, etc. Al carecer de convenio propio se recurre al de otros ámbitos que no están adecuados a nuestras realidades (oficinas y despachos, ocio, intervención social, artes gráficas...). Recurrir a marcos regulatorios no adecuados dificulta tanto la correcta valoración de costes como la selección del personal y acaba propiciando situaciones que perjudican a todos los agentes implicados:

- contratadores de origen (muchas administraciones, sobre todo las locales);

- contratadores de intermediación (empresas de servicios);

- profesionales de la información;

- la ciudadanía como destinataria final de los servicios.

Tomar conciencia del problema y conseguir visibilidad como sector es fundamental para intentar negociar un marco propio que se adapte a nuestra idiosincrasia.

Para crear o modificar un convenio laboral es necesario que sindicatos y empresas lleguen a un acuerdo. Es cierto que donde se ha producido concentración de trabajadores (BNE, Azkuna, Ubik o la Red de Bibliotecas de Las Palmas) las presiones sindicales han forzado nuevas condiciones que han mejorado situaciones (jornadas excesivas, categorías laborales específicas, salarios muy bajos, carencia de pluses...) pero sin solucionar el problema de fondo: la carencia de un marco de referencia laboral propio.

Somos un colectivo que no concentra gran número de trabajadores en un mismo centro, la tasa de temporalidad es muy elevada y carecemos de un tejido empresarial significativo por lo que habitualmente estamos infrarrepresentados en sindicatos y organizaciones empresariales y no participamos en las negociaciones. Como sector (los profesionales a través de los sindicatos y las empresas como colectivo con personalidad propia) necesitamos ganar representatividad para dar

"Como sector (los profesionales a través de los sindicatos y las empresas como colectivo con personalidad propia) necesitamos ganar representatividad para dar a conocer las particularidades de nuestros perfiles profesionales para que sean tenidos en cuenta en la redacción del marco laboral" 
aconocer las particularidades de nuestros perfiles profesionales para que sean tenidos en cuenta en la redacción del marco laboral. Es un movimiento que debemos realizar como sector, al unísono, porque de la misma manera que no se reconoce a los profesionales, tampoco se visualiza la existencia de un conjunto empresarial definido y reconocido como interlocutor válido. En las asociaciones creemos que debemos conseguir que nuestras reivindicaciones sean atendidas por los sindicatos y que las empresas deben unirse para no ser diluidas entre las más potentes y generalistas a las que no les conviene definir ni perfilar el nicho de mercado.

\section{La contratación pública socialmente responsable}

La Comisión Europea ha publicado un informe (Comisión Europea, 2020) que recoge casos de buenas prácticas sobre cómo los compradores públicos han implementado la contratación pública socialmente responsable (SRPP, Socially responsible public procurement), con el fin de promover oportunidades de empleo, trabajo decente, inclusión social, accesibilidad, comercio ético, diseño para todos, a la vez que se logra un mayor cumplimiento de los estándares sociales. Adoptar criterios de compra pública socialmente responsable exige a los compradores que miren más allá del precio y la calidad de los productos o servicios que desean adquirir y que también consideren cómo se produce y obtiene un producto o servicio. Al utilizar su poder adquisitivo para optar por bienes y servicios que generen resultados sociales positivos, pueden hacer una contribución importante al desarrollo sostenible. Tanto el sector público como el privado reconocen cada vez más la necesidad de abordar los tres pilares de la sostenibilidad (social, ambiental y económico) en las adquisiciones. La contratación pública socialmente responsable influye en el mercado en general tanto en el lado de la demanda como en el de la oferta. Fesabid considera prioritario concienciar a los responsables políticos sobre esta realidad.

Las directivas de contratación pública de 2014 (Unión Europea, 2014) dejan claro que los aspectos sociales pueden tenerse en cuenta a lo largo del ciclo de contratación, desde la consulta preliminar del mercado, pasando por el uso de reservas y el régimen ligero, hasta los criterios de adjudicación social y las condiciones de ejecución del contrato. Los compradores públicos de toda Europa están comenzando a aprovechar estas oportunidades y a demostrar un impacto social real en sus compras. A pesar de ello, los Estados miembros aún no están explotando plenamente las posibilidades de la contratación pública como herramienta estratégica para apoyar los objetivos de la política social.

\section{Acciones emprendidas desde Aldee: hacia un modelo de contratación pública socialmente responsable}

Gipuzkoa es un territorio en el que las externalizaciones han sido traumáticas y todos los agentes implicados son conscientes de la necesidad de buscar soluciones de futuro. Aldee, la asociación profesional de Euskadi, combina su conocimiento de la situación con su posición equidistante y neutral entre los diferentes protagonistas y su iniciativa de puesta en marcha de un proceso piloto para establecer un convenio sectorial que garantice una contratación pública en igualdad de condiciones para todas las partes ha sido bien acogida por todos los agentes implicados. Las acciones realizadas hasta el momento han sido:

- reunión con todos los sindicatos para exponerles la necesidad de un convenio colectivo propio y solicitar su ayuda;

- invitar a las empresas del sector a juntarse para no ser diluidas entre las más potentes y generalistas a las que no les conviene definir ni perfilar el nicho de mercado3;

- poner en contacto a empresas y sindicatos para afrontar la negociación del convenio;

- exponer a las administraciones la necesidad de establecer medidas correctoras en sus pliegos de condiciones y explicar los beneficios de un marco regulatorio propio que garantice una contratación pública socialmente responsable.

La experiencia de Aldee en Gipuzkoa servirá de modelo para futuras actuaciones lideradas por Fesabid.

\section{Fesabid y Aldee: hacia un modelo de contratación pública socialmente responsable}

Desde las asociaciones y colegios profesionales podemos facilitar la interlocución entre administraciones, empresas, profesionales y sindicatos para construir un modelo de externalización que fortalezca el sector de la información en España, y por eso en Fesabid se ha constituido un grupo de trabajo interno que ha iniciado las siguientes acciones:

- análisis de los distintos convenios colectivos a los que se adscriben los profesionales contratados de nuestro sector para conocer la situación real; 
- valoración de la adecuación de dichos convenios a nuestra realidad profesional y acordar, si se considera necesario, negociar un convenio propio;

- establecimiento de contactos con los agentes implicados en una posible futura negociación: sindicatos, empresas del sector, trabajadores;

- acompasar las actuaciones al ritmo de las negociaciones colectivas para aprovechar los momentos en los que es posible incidir en los convenios porque se encuentran vencidos;

- interlocución con las administraciones para exponer las ventajas de la contratación pública socialmente responsable.

Veremos.

\section{Notas}

1. Muchas veces nos preguntamos para qué sirven las jornadas y los congresos profesionales. Creo (espero que los comités científicos no se enfaden), que lo mejor de esas reuniones profesionales es su carácter social, esos encuentros cotidianamente improbables que propician.

http://www.fesabid.org/barcelona2019

2. Con la recesión económica de 2008 el número de externalizaciones en el ámbito municipal español ha aumentado, pero sin la existencia de registros oficiales sobre el tema es complejo analizar el fenómeno.

3. Gracias a la iniciativa de Aldee se acaba de constituir Galde: Asociación Empresarial de Archiveros, bibliotecarios y Centros de Documentación de Gipuzkoa. Entre sus objetivos:

"fomentar la calidad del trabajo que, por diferentes motivos, se está difuminando cada vez más debido a la degradación conceptual y laboral. Esta situación ha generado un deterioro de las condiciones de trabajo, intrusismo, deslocalización, escasa profesionalidad... En la Asociación Empresarial trabajaremos por el fortalecimiento de la profesionalidad y la mejora de las condiciones laborales en nuestro territorio".

http://www.ereiten.eus/berria/galde-gipuzkoako-artxibozain-liburuzain-eta-dokumentazio-guneen-enpresaburuen-elkarteal

\section{Referencias}

Andalucía (2017. "Resolución del Defensor del Pueblo Andaluz formulada en la queja 15/5470 dirigida a Consejería de Hacienda y Administración Pública". Defensor del Pueblo Andaluz 28 julio.

https://www.defensordelpuebloandaluz.es/reclamamos-el-establecimiento-de-criterios-objetivos-para-laexternalizacion-del-sector-publico

Comisión Europea (2020). Making socially responsible responsible public procurement work. 71 good practice cases. https://op.europa.eu/en/publication-detail/-/publication/69fc6007-a970-11ea-bb7a-01aa75ed71a1

Euskadi (2019). Pliego de cláusulas administrativas particulares que habrá de regir la licitación, mediante procedimiento abierto, para la contratación del servicio de atención en sala en Ubik, espacio de biblioteca de creación de centro internacional de cultura contemporánea, S.A.

https://www.contratacion.euskadi.eus/w32-kpeperfiles/contenidos/anuncio_contratacion/expjaso23236/es_doc/ adjuntos/clausulas_admin_particulares1.pdf?rand $=84718$

Euskadi (2021). "Organización y descripción de la documentación contemporánea de los archivos históricos de la Diputación Foral de Gipuzkoa, dividido en dos lotes". Plataforma Contratación Pública en Euskadi.

https://www.contratacion.euskadi.eus/w32-kpeperfi/es/contenidos/anuncio_contratacion/expjaso31688/es_doc/es_ arch_expjaso31688.htm/?ruta=http://www.contratacion.euskadi.eus/w32-kpeperfiles

Juárez-Urquijo, Fernando (2019). "Algunas reflexiones sobre el marco de contratación en bibliotecas públicas". Anuario ThinkEPI, v. 13, e13b04.

https://doi.org/10.3145/thinkepi.2019.e13b04

Tabakalera (2019). "Ubik reabre sus puertas a la ciudadanía". Tabakalera, 7 agosto.

https://www.tabakalera.eus/es/ubik-reabre-sus-puertas-la-ciudadania

Unión Europea (2014). "Directiva 2014/23/UE del Parlamento Europeo y del Consejo, de 26 de febrero de 2014 relativa a la adjudicación de contratos de concesión". Diario Oficial de la Unión Europea, 28 marzo.

https://eur-lex.europa.eullegal-content/ES/TXT/PDF/?uri=CELEX:32014L0023\&from=EN

Fernando Juárez-Urquijo

ferjur@gmail.com 


\section{Hablemos \\ Sofía Martins}

Enhorabuena por este excelente artículo, Fernando, nos aportas una reflexión tan urgente como necesaria. A menudo silenciosa.

Hablemos, entonces, dialoguemos entre todas las partes, sobre la responsabilidad social en las contrataciones públicas, y el papel de las asociaciones en la regulación de nuestros ecosistemas profesionales. De la ética y de la deontología en la praxis laboral de las contrataciones.

Voy a aportar mi granito de arena, a mi manera.

\section{Yo pertenezco a una de las partes involucradas}

Como bibliotecaria de fondos antiguos toda mi vida laboral he participado en proyectos externalizados en varias instituciones públicas, con diferentes tipos de contratos. En España y en Portugal. Siempre me ha parecido aliciente participar en ellos, por varios motivos que no vienen al caso (o sí).

Sin embargo, mi percepción es la de estar eligiendo mi recorrido laboral en una no-igualdad de condiciones con otros compañeros. No es un sistema equitativo.

Creo y me reveo en un servicio público, en la función social de mi trabajo dentro del Estado. Las oposiciones son otra vía que yo pondero. Pero os seré honesta: a mí se me presentan más como una huida de la precariedad que como una elección vocacional. (Si el miembro de un tribunal me está leyendo...). O sea, una cosa es elegir, trazar una meta, otra es huir a falta de pan o al cansancio y al desánimo...

\section{A esto vamos...}

Un trabajo externalizado es sinónimo de precariedad laboral. Los sabemos muchos de nosotros aquí. Y este será el futuro de tantos nuevos compañeros, como describe Fernando.

\section{Preguntas, metafísicas...}

Si lo sabemos ¿por qué no lo hablamos más y de manera articulada? No lo pregunto desde la simpleza. ¿Cómo nos vemos y construimos profesionalmente (en este contexto)? ¿Buscamos las asociaciones, somos individualistas? ¿Incluimos a, conversamos con, los técnicos de las instituciones que muchas veces desconocen nuestra realidad? ¿Nos sentimos sus pares? ¿Cómo dialogamos con las empresas sujetas al mismo juego?

Claro que nada de esto es ajeno a nuestro momento histórico político y económico, a una cultura y mentalidad.

Asumámoslo, es un tema complejo. De variables múltiples, empezando por la vida de cada uno. Y, a menudo, las reglas del juego parecen ser inmutables e incuestionables. ¿Será así?

\section{Concluyendo... Datos (más o menos empíricos y verificables y sujetos a discusión)}

En estos años he podido vivir y observar cómo las decisiones sobre las licitaciones de las externalizaciones iban cambiando. A montante. A montante, por parte de quien decidía cómo licitar y cuáles los criterios de selección. Esto es un dato importante, en mi opinión.

Gradualmente se ha pasado de valorar el lado técnico a poner en relieve el lado económico, no sin ciertos riesgos para la calidad final del trabajo, muy a pesar de las partes involucradas.

He podido constatar cómo bibliotecarios, archiveros y documentalistas han sido alejados de procesos decisorios, que se han trasladado a las manos de gestores/administrativos que, en general, desconocen nuestra labor y cultura técnica científica.

Los sueldos... son como barca en tierra seca -sin oleaje-, lejos de espejar nuestra formación y experiencia. ¿Por qué no definir requisitos de mínimos salariales y condiciones laborales en las licitaciones? Pregunto.

\section{Nosotros/as, las asociaciones}

¿Qué voz, qué visibilidad, tenemos nosotros profesionales -me refiero a todos- en lo que nos concierne? ¿Cómo se ve nuestro trabajo especializado, técnico cultural científico social, desde la administración? ¿Creemos que esta cuestión aquí abordada es algo marginal? Es el destino, lo que nos toca vivir... Bueno, hasta los griegos han desafiado a sus dioses...

\section{Necesitamos a las asociaciones en este proceso}

Pienso que hablar de este tema es dar visibilidad a nuestra profesión como un todo, es valorar nuestras competencias, es definir perfiles profesionales, condiciones equitativas de trabajo. $Y$ también, es abrir camino para que haya profesionales que quieran y puedan elegir dignamente sus caminos. 
Un juego de varias partes, y complejo. Asumo la responsabilidad en lo que me corresponde, y por eso respondo aquí públicamente a Fernando, asumiendo sus consecuencias.

Dialoguemos.

Sofía Martins

sophia.msimoes@gmail.com

\section{*** \\ El papel de la Universidad José-Antonio Gómez-Hernández}

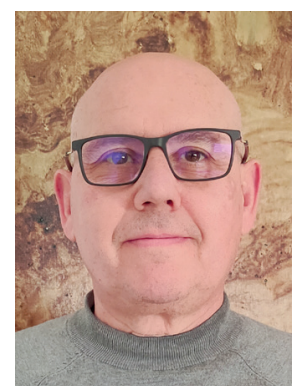

Fernando hace un diagnóstico preciso y muy necesario -diría que imprescindible- de la precariedad laboral en el sector GLAM, que afecta a los servicios externalizados y también al propio sector público, donde en muchas ocasiones los puestos se convocan en condiciones inferiores a la cualificación necesaria para el ejercicio profesional, o con requisitos y contenidos obsoletos.

Así lo viene reflejando el Observatorio de Defensa de la Profesión de Fesabid, que recibe numerosísimas denuncias de situaciones y convocatorias inaceptables para la dignidad profesional.

https://www.fesabid.org/en-accion/observatorio

Poco se puede añadir a lo que se ha señalado: la carencia de convenios propios y el que la contratación pública no atienda en ocasiones a criterios socialmente responsables genera condiciones de externalización que expulsa de nuestros ámbitos profesional a muchas personas bien cualificadas, o los precariza si no tienen alternativas mejores. Diría también que las malas condiciones laborales y las bajas expectativas disuaden a bastantes jóvenes, cuando tienen que elegir sus estudios al dirigirse a la universidad, de optar por los grados universitarios relacionados con nuestra profesión. Y por ello podemos estar perdiendo muchas vocaciones brillantes que contribuirían al relevo generacional y enriquecerían nuestra profesión si vieran más factible incorporarse a ella.

Es muy necesario luchar por la protección de los derechos laborales en nuestra profesión para quienes actualmente la ejercen, porque eso la hará más atractiva para quienes aspiren a estudiarla y a desempeñarla en el futuro. La racionalidad neoliberal imperante en el mercado laboral encamina a una mayor precariedad en general, y particularmente en nuestro sector, y eso lo deberíamos abordar con pautas como las que Fernando propone hacer llegar a las administraciones, los sindicatos y las empresas del sector. Incluyendo la reivindicación cuando sea necesario y factible. También necesitamos que universidades y asociaciones vayamos muy de la mano para fortalecernos mutuamente, porque una profesión tiene más fortaleza si tiene una o varias titulaciones de referencia fuertes. Nuestra labor es muy complementaria.

Una anécdota: al poco de empezar a trabajar como auxiliar de biblioteca (lo que hoy sería C1) en mi universidad, en todas las universidades se vio que era justo que promocionáramos a la categoría de ayudante (A2), para homologarnos con los colegas que hacían funciones parecidas en el Ministerio de Cultura. Era mediados de los años ochenta, y en un breve plazo se hizo, haciéndonos sentir reconocidos y tratados justamente como profesionales. Muchos años después diría que la situación profesional en general ha ido para atrás especialmente para las nuevas generaciones. Y revertir esto es una prioridad para el futuro profesional y para la dignidad de una profesión que en todas sus variantes debe ejercerse de acuerdo con la relevancia del derecho a la información. Reivindiquemos esto hoy que es el Día internacional del derecho a saber. 


\section{Observatorio de Defensa de la Profesión Ana Bernardo-Suárez}

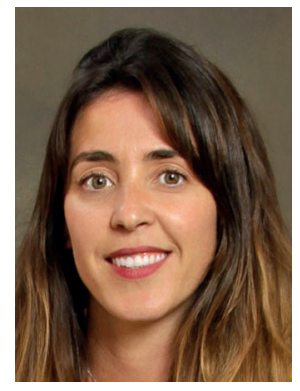

Fernando, gracias por visibilizar y reflexionar sobre el escenario laboral en GLAM, en el que la externalización de servicios está adquiriendo un mayor protagonismo y la precariedad laboral está a la orden del día, incluido el sector público. Es muy interesante ir acercándose al modelo de contratación pública responsable descrito en las directivas de la Unión Europea que mencionas y que el ordenamiento jurídico español ya aplica través de la Ley 9/2017, de 8 de noviembre, de contratos del sector público (España, 2017), reconociendo la obligación de la incorporación de cláusulas sociales y medioambientales. Como buena práctica me gustaría mencionar la Guía práctica para la inclusión de cláusulas de responsabilidad social y medioambiental en la contratación administrativa de la administración del Principado de Asturias y su sector público (Asturias, 2018) aunque ciertamente muchas veces no se aplica en la contratación en la administración asturiana.

Al hilo de lo que comenta José Antonio, y como coordinadora del Observatorio de Defensa de la Profesión de Fesabid, me gustaría difundir a través de este canal las diferentes iniciativas llevadas a cabo por el Observatorio desde su reciente creación en 2021.

https://www.fesabid.org/en-accion/observatorio/

El Observatorio nace del grupo de trabajo de Defensa de la profesión, grupo estable dentro de la estructura de Fesabid, y una línea estratégica de trabajo de la Federación. Uno de sus primeros trabajos fue la realización del "Mapa de situación de la defensa de la profesión en las Asociaciones y Colegios Profesionales" (Fesabid, 2019). Además de los resultados del mismo, como la visibilización de la tarea de las asociaciones/colegios en el tema de defensa de la profesión, en el que se demostró una base asociativa muy activa y preocupada por la realidad que afecta a la profesión, una línea de acción propuesta fue la creación de un "Observatorio de Defensa de la Profesión".

El Observatorio de la defensa de la profesión es ya una realidad en el marco de trabajo de cooperación y colaboración entre Fesabid y las asociaciones y colegios profesionales miembros y, tras un año de actividad, se han realizado actividades como:

- Visibilización en redes sociales de ofertas poco dignas que nos hacen llegar los profesionales.

https://www.fesabid.org/denuncia-desde-el-observatorio-de-defensa-de-la-profesion

- Reivindicación profesional para la Biblioteca Regional de Murcia.

https://www.fesabid.org/reivindicacion-biblioteca-regional-murcia

La siguiente acción prevista del Observatorio es crear un espacio de reflexión conjunta, en el que se considera que la profesionalización del sector es un tema clave sobre el que deliberar con los agentes relacionados con el ejercicio de la profesión. Se materializará a través del II Foro de Reflexión del Sector de la Información y la Documentación el próximo mes de noviembre. Este Foro nos ayudará a observar la situación en la que nos encontramos con una mirada amplia y con perspectiva de futuro.

Actualmente estamos trabajando en su organización y os agradecemos nos hagáis llegar cualquier aportación/sugerencia que consideréis oportunas.

Os iremos informando sobre su avance.

\section{Referencias}

Asturias (2018). Guía práctica para la inclusión de cláusulas de responsabilidad social y medioambiental en la contratación administrativa de la administración del Principado de Asturias y su sector público.

https://sede.asturias.es/Proveedores/FICHEROSIESTRUCTURA\%202011/NORMATIVA\%20PERFILIINSTRUCCIONES\% 20 LEY\%209_2017/guia_practica_clausulas_social_y_m_amb_07-05-2018.pdf

España (2017). "Ley 9/2017, de 8 de noviembre, de contratos del sector público, por la que se transponen al ordenamiento jurídico español las Directivas del Parlamento Europeo y del Consejo 2014/23/UE y 2014/24/UE, de 26 de febrero de 2014". BOE, n. 272, 9 noviembre.

https://www.boe.es/buscarlact.php?id=BOE-A-2017-12902

Fesabid (2019). Mapa de situación de la defensa de la profesión en las Asociaciones y Colegios Profesionales. Madrid: Fesabid.

https://www.fesabid.org/wp-content/uploads/2021/04/mapadefensa051119.pdf 